\title{
SHEAR INSTABILITIES FOR THE BUCKLING OF NONLINEARLY ELASTIC PLATES
}

\author{
BY \\ PABLO V. NEGRON-MARRERO \\ University of Puerto Rico, Rio Piedras, Puerto Rico
}

\begin{abstract}
In this work we apply singularity theory and global bifurcation theory to the problem of the axisymmetric buckling of circular plates. We show that a certain shear parameter can be used as an unfolding parameter and we get detailed local and global structure of the solution set. We get conditions for a singular point to be of codimension one.
\end{abstract}

1. Introduction. Usually the problems arising in the physical sciences and engineering involve several additional parameters that characterize certain properties of the system. The study of parameter-dependent problems has a long history which we shall not attempt to describe here. We mention however the very important works of Leray and Schauder [13], Crandall and Rabinowitz [7], and Rabinowitz [18] for oneparameter problems. More recently these results have been generalized to problems depending on any finite number of parameters by Alexander and Antman [1] and to the case of infinite-dimensional parameter problems by Alexander and Antman [2]. All of these works give global results and with additional conditions one can get detailed local results.

Quite often symmetries in the physical system induce corresponding symmetries in the equilibrium equations. These can be used to get further qualitative information on the solutions via singularity theory or by a group-theoretic approach. (See Sattinger [19] and Golubitsky and Schaeffer [8]. See also Healey [9, 10] for a survey and a general framework in which symmetry is used in elastostatics.) Although the usual results in singularity theory are local they can be made global by combining them with global bifurcation theory (see Antman and Pierce [4]). In this work we apply singularity theory and global bifurcation theory to the problem of axisymmetric buckling of circular plates. Our results are similar in spirit to those of [4] but the actual development is considerably more difficult due to anisotropy and the singular behavior of the equations at the center of the plate.

In Sec. 2 we introduce the fifth-order system of ordinary differential equations describing the axisymmetric buckling of circular plates. In Sec. 3 we summarize the results concerning the existence of trivial (unbuckled) states of the plate. Section 4 
is devoted to the analysis of the linearization of the equilibrium equations of Sec. 2 about the trivial state of Sec. 3. We give conditions under which the linearized equations can be transformed into a regular boundary value problem (thus making the singularity at the center of the plate not so severe). In Sec. 5 we show that a certain monotonicity condition (cf. (2.10c)) allows for the use of the implicit function theorem to reduce our fifth-order system to a second-order boundary value problem. By generalizing the development of Sec. 4, we transform this singular second-order equation to one which is regular. Then in Sec. 6 we emply a shooting method to reduce the question of the existence of solutions of the second-order boundary value problem to that of finding the roots of a scalar equation. We then show that certain shear parameter can be used as an unfolding parameter and we get detailed local and global structure of the solution set. In particular, we get conditions for a singular point to be of codimension one. The physical interpretation of this is that if the plate is subjected to a gradually increasing pressure on its edge, its diameter will shorten but the plate does not lose it straightness by buckling because there are no nearby equilibrium states. However, if the thrust is large enough, then a large transverse force can move the compressed straight plate to a stable large buckled configuration where it will remain after the transverse force is removed. In Sec. 7 we give two examples of this sort of phenomena for certain classes of materials.

Notation. $R$ denotes the set of real numbers. We denote by $\|\cdot ; X\|$ the norm of the normed linear space $X$. For any function $u:[0,1] \rightarrow R$, we denote by $u^{(k)}$ its $k$ th derivative. We let

$$
\begin{gathered}
C^{k}[0,1]=\left\{u:[0,1] \rightarrow R \mid u^{(j)} \text { is continuous in }[0,1], 0 \leq j \leq k\right\}, \\
\left\|u ; C^{k}[0,1]\right\|=\max _{0 \leq j \leq k} \max _{0 \leq x \leq 1}\left|u^{(j)}(x)\right| .
\end{gathered}
$$

We use the notation

$$
f(s) \sim g(s) \text { as } s \rightarrow 0^{+},
$$

which means that $f(s) / g(s) \rightarrow 1$ as $s \rightarrow 0^{+}$. We shall denote the end of a proof, theorem, definition, etc. by the symbol.

2. Formulation of the governing equations. We introduce here the equations which describe the deformations of nonlinearly elastic plates. For a full derivation we refer to Antman [3] and Negron-Marrero and Antman [17].

We consider an axisymmetric deformation of a circular plate of radius one in its reference configuration. The strains for this problem are given by

$$
w(s)=(t(s), n(s), h(s), z(s), m(s)) .
$$

(Here the variable $s$, which belongs to $[0,1]$, measures distance to the center of the plate in the reference configuration.) The strains $t(s)$ and $n(s)$ measure the elongation of circumferential and radial fibers respectively, while $z(s)$ and $m(s)$ measure their bendings, and $h(s)$ is a measure of shear. The requirement that an infinitesimal volume in the reference configuration cannot be reduced to a point during the deformation leads to the inequalities (see [3])

$$
t(s)>c|z(s)|, \quad n(s)>c|m(s)|,
$$


for some positive constant $c$. We denote by $W$ the set of all $w$ 's satisfying (2.2). $W$ is convex. If we define $a(s)$ by

$$
a^{\prime}(s)=m(s)
$$

then one gets (see [3])

$$
\begin{gathered}
(s t(s))^{\prime}=n(s) \cos a(s)-h(s) \sin a(s), \\
z(s)=(\sin a(s)) / s,
\end{gathered}
$$

where the prime denotes differentiation with respect to $s$. The stresses corresponding to $(2.1)$ are

$$
\left(T^{*}(s), N^{*}(s), H^{*}(s), Z^{*}(s), M^{*}(s)\right) .
$$

If the plate is subjected to a normal pressure of $q$ units of force (per reference length) on the edge $s=1$, then the equilibrium equations for the plate are given by

$$
\begin{aligned}
\left(s N^{*}(s)\right)^{\prime}= & s H^{*}(s) m(s)+T^{*}(s) \cos a(s), \\
\left(s H^{*}(s)\right)^{\prime}= & -s N^{*}(s) m(s)-T^{*}(s) \sin a(s), \\
\left(s M^{*}(s)\right)^{\prime}= & s\left(N^{*}(s) h(s)-H^{*}(s) n(s)\right) \\
& +Z^{*}(s) \cos a(s), \quad 0<s<1,
\end{aligned}
$$

with boundary conditions

$$
\begin{gathered}
a(0)=0=a(1), \\
\left.s t(s)\right|_{s=0^{+}}=0, \\
H^{*}(0)=0, \quad N^{*}(1)=-q .
\end{gathered}
$$

Constitutive equations. We assume that the material of the plate is homogeneously elastic by requiring that there exist thrice continuously differentiable functions

$$
T, N, H, Z, M: W \rightarrow R,
$$

such that

$$
T^{*}(s)=T(w(s)), \text { etc. } \quad(\text { cf. }(2.1)) .
$$

The strong ellipticity condition from three-dimensional elasticity implies that the functions (2.8) satisfy the following monotonicity conditions:

$$
\left[\begin{array}{lll}
N_{n} & N_{h} & N_{m} \\
H_{n} & H_{h} & H_{m} \\
M_{n} & M_{h} & M_{m}
\end{array}\right],\left[\begin{array}{ll}
T_{t} & T_{z} \\
Z_{t} & Z_{z}
\end{array}\right] \text { are positive-definite }
$$

(see [3]). We assume also that

$$
\left[\begin{array}{ccc}
N_{n} & N_{h} & N_{t} \\
H_{n} & H_{h} & H_{t} \\
T_{n} & T_{h} & T_{t}
\end{array}\right] \text { is positive-definite. }
$$

This condition, which is not a consequence of the strong ellipticity condition, avoids certain kinds of necking instabilities that could occur if part of the plate is in sufficiently large tension (cf. Negron-Marrero [16]). 
We require that the functions (2.8) satisfy the following symmetry conditions which ensure that deformed states come in mirror images:

$$
\begin{gathered}
T, N, Z, M \text { are even in } h, \\
H \text { is odd in } h, \\
T(t, n, h,-z,-m)=T(t, n, h, z, m), \\
N(t, n, h,-z,-m)=N(t, n, h, z, m), \\
H(t, n, h,-z,-m)=H(t, n, h, z, m), \\
Z(t, n, h,-z,-m)=-Z(t, n, h, z, m), \\
M(t, n, h,-z,-m)=-M(t, n, h, z, m) .
\end{gathered}
$$

We impose the following growth conditions:

$$
\begin{array}{ll}
T \rightarrow-\infty & \text { as } t-c|z| \rightarrow 0^{+}, \\
T \rightarrow \infty & \text { as } t \rightarrow \infty, \\
N \rightarrow-\infty & \text { as } n-c|m| \rightarrow 0^{+}, \\
N \rightarrow \infty & \text { as } n \rightarrow \infty, \\
H \rightarrow \pm \infty & \text { as } h \rightarrow \pm \infty, \\
Z \rightarrow \pm \infty & \text { as } z \rightarrow \pm \infty, \\
M \rightarrow \pm \infty & \text { as } m \rightarrow \pm \infty .
\end{array}
$$

For simplicity we assume that the reference configuration is stress-free so that

$$
N(1,1,0,0,0)=0=T(1,1,0,0,0) .
$$

Equations (2.3)-(2.7) define a fifth-order system of differential equations for the variables $(t, n, h, a)$, which we call our boundary value problem. We seek solutions that satisfy the strict inequalities (2.2).

3. Trivial solutions. The trivial solutions of our problem correspond to the unbuckled states of the plate. These are defined by the relations

$$
h=0=a .
$$

In this case, with $r(s)=s t(s)$, our boundary value problem reduces to

$$
\begin{gathered}
\left(s N\left(r(s) / s, r^{\prime}(s)\right)\right)^{\prime}=T\left(r(s) / s, r^{\prime}(s)\right), \quad 0<s<1, \\
r(0)=0, \quad N\left(r(1), r^{\prime}(1)\right)=-q, \\
r(s) / s, \quad r^{\prime}(s)>0, \quad 0 \leq s \leq 1,
\end{gathered}
$$

where for simplicity we write $T(t, n)$ instead of $T(t, n, 0,0,0)$, etc. We say that the material of the plate is azimuthally reinforced at $t$ if

$$
N_{n}(t, t)+N_{t}(t, t)<T_{n}(t, t)+T_{t}(t, t) .
$$

From the results of Antman and Negron-Marrero [5, 17 (Sec. 3, Theorem 2)] we get the following result concerning the existence of solutions of the boundary value problem (3.2), (3.3). 
TheOREM (3.1). Let (3.4) hold and let the function $N$ satisfy

$$
N_{n}(t, n)+N_{t}(t, n)>0 \quad \text { for all } t, n,
$$

and

$$
\begin{aligned}
N(t, n) \rightarrow-\infty & \text { as } t \rightarrow 0^{+} \text {or } n \rightarrow 0^{+} \\
& \text {and } t^{2}+n^{2}<K^{2},
\end{aligned}
$$

for each fixed constant $K$. Hence for each $q \geq 0$ the boundary value problem (3.2), (3.3) has a solution $r$ in $C^{1}[0,1]$ that satisfies $(3.3)$ with $r^{\prime}(0)=1$. If, in addition, $N$ and $T$ satisfy

$$
\left[\begin{array}{ll}
N_{n} & N_{t} \\
T_{n} & T_{t}
\end{array}\right] \text { is positive-definite }
$$

(which follows from $(2.10 \mathrm{c})$ ), then the solution is unique. If we strengthen condition (3.4) to

$$
k\left(N_{n}(t, t)+N_{t}(t, t)\right)<k T_{n}(t, t)+T_{t}(t, t),
$$

$k \geq 2$, then $r$ is $C^{k}[0,1]$ and

$$
r^{(j)}(0)=0, \quad 2 \leq j \leq k .
$$

To emphasize the dependence of $r$ on $q$ we sometimes will write $r(s, q)$.

4. The linearized equations. Let

$$
w_{0}(s, q)=\left(r(s, q) / s, r^{\prime}(s, q), 0,0,0\right) .
$$

For any constitutive function or its partial derivatives we write

$$
N^{0}(s, q)=N\left(w_{0}(s, q)\right) \text {, etc. }
$$

Hence the linearization of the boundary value problem of Sec. 2 about the trivial solution (4.1) is given by (see [17])

$$
\begin{gathered}
\left(s M_{m}^{0}(s, q) p^{\prime}(s)+M_{z}^{0}(s, q) p(s)\right)^{\prime} \\
=s N^{0}(s, q)\left[r^{\prime}(s, q)-N^{0}(s, q) / H_{h}^{0}(s, q)\right] p(s) \\
+Z_{z}^{0}(s, q) p(s) / s+Z_{m}^{0}(s, q) p^{\prime}(s), \\
p(0)=0=p(1),
\end{gathered}
$$

where $p$ corresponds to variations in the function $a$. The existence of solutions of this boundary value problem has been established by Negron-Marrero and Antman [17] by comparison methods. However we proceed here differently by transforming the singular operator in (4.3a) into a regular one. This, apart from automatically giving results on the existence of eigenvalues and eigenfunctions for (4.3), will provide us with a more natural approach (from the point of view of singularity theory) to deal with the nonlinear boundary value problem of Sec. 2 . The essential hypothesis in this analysis will be condition (3.8). 
If we expand the derivatives in the left side of $(4.3 \mathrm{a})$, then we get the equivalent equation

$$
p^{\prime \prime}(s)+R(s, q) p^{\prime}(s) / s+Q(s, q) p(s) / s^{2}=0,
$$

where

$$
\begin{gathered}
R(s, q)=\left[\left(s M_{m}^{0}(s, q)\right)^{\prime}+M_{z}^{0}(s, q)-Z_{m}^{0}(s, q)\right] / M_{m}^{0}(s, q), \\
Q(s, q)=\left[s M_{z}^{0}(s, q)^{\prime}-s^{2} N^{0}(s, q)\left(r^{\prime}(s, q)\right.\right. \\
\left.\left.\quad-N^{0}(s, q) / H_{h}^{0}(s, q)\right)-Z_{z}^{0}(s, q)\right] / M_{m}^{0}(s, q) .
\end{gathered}
$$

The transformation

$$
p(s)=s^{v} u(s),
$$

takes equation (4.4) into

$$
\begin{gathered}
u^{\prime \prime}(s)+[2 v+R(s, q)] u^{\prime}(s) / s+C(s, q) u(s) / s^{2}=0, \\
C(s, q)=v(R(s, q)-R(0, q))+Q(s, q)-Q(0, q),
\end{gathered}
$$

provided $v$ satisfies

$$
v(v-1)+v R(0, q)+Q(0, q)=0 .
$$

Furthermore with

$$
\Phi(s, q)=\exp \left[\int[(2 v+R(s, q)) / s] d s\right],
$$

we get that

$$
\left(\Phi(s, q) u^{\prime}(s)\right)^{\prime}+\Phi(s, q) C(s, q) u / s^{2}=0 .
$$

Define now

$$
\begin{gathered}
f(s, q)=1+\int_{s}^{1}(1 / \Phi(t, q)) d t, \\
\Theta(s, q)=\Phi(s, q) f^{2}(s, q), \\
c(s, q)=-C(s, q) f^{2}(s, q) \Phi(s, q) / s^{2} .
\end{gathered}
$$

We now can prove the following:

THEOREM (4.1). The eigenvalues of the BVP (4.3) coincide with those of

$$
\begin{gathered}
-\left(\Theta(s, q) U^{\prime}(s)\right)^{\prime}+c(s, q) U(s)=0, \quad 0<s<1, \\
U(0)=0=U(1) .
\end{gathered}
$$

If $U\left(s, q^{*}\right)$ is an eigenfunction of (4.12) with corresponding eigenvalue $q^{*}$, then

$$
p\left(s, q^{*}\right)=s^{\gamma} f\left(s, q^{*}\right) U\left(s, q^{*}\right)
$$

is an eigenfunction of (4.3) with corresponding eigenvalue $q^{*}$, where $\gamma$ is the positive root of (4.8). Moreover if (3.8) holds with $k \geq 2$, then the BVP (4.12) is regular provided

$$
\begin{aligned}
& b^{2}+4 T_{t}^{0}(0, q) / N_{n}^{0}(0, q)<(k-1)^{2}, \\
& b=\left(N_{t}^{0}(0, q)-T_{n}^{0}(0, q)\right) / N_{n}^{0}(0, q) .
\end{aligned}
$$


Proof. The statement about the equivalence of the boundary value problems (4.3) and (4.12), and relation (4.13) follow from (4.11) and the results in Kaper, Kwong, and Zettl [12].

From the definitions (4.5) and Theorem (3.1) with $k \geq 2$, we get that

$$
\begin{gathered}
R(0, q)=1+\left(M_{z}^{0}(0, q)-Z_{m}^{0}(0, q)\right) / M_{m}^{0}(0, q), \\
Q(0, q)=-Z_{z}^{0}(0, q) / M_{m}^{0}(0, q) .
\end{gathered}
$$

(Note that since $r^{\prime}(0, q)=1$ by Theorem (3.1), then $\gamma$ as defined by (4.8) is independent of $q$ as well as $b$ in (4.14).) The results in [3, Sec. 10, Eqs. (10.52) and (10.53)] show that there exists a positive function $d_{0}(s)$ such that

$$
\begin{aligned}
& \left(Z_{z}^{0}(s, q), Z_{m}^{0}(s, q), M_{z}^{0}(s, q), M_{m}^{0}(s, q)\right) \\
& \quad \quad=d_{0}(s)\left(T_{t}^{0}(s, q), T_{n}^{0}(s, q), N_{t}^{0}(s, q), N_{n}^{0}(s, q)\right) .
\end{aligned}
$$

Using $(4.15 \mathrm{c})$ in $(4.15 \mathrm{a}, \mathrm{b})$ we get that

$$
R(0, q)=1+b, \quad Q(0, q)=-T_{t}^{0}(0, q) / N_{n}^{0}(0, q) .
$$

Thus, from (4.8) we get that

$$
\gamma=\left(-b+\left(b^{2}+4 T_{t}^{0}(0, q) / N_{n}^{0}(0, q)\right)^{1 / 2}\right) / 2 .
$$

Let

$$
\begin{aligned}
\beta & =2 \gamma+1+b \\
& =1+\left(b^{2}+4 T_{t}^{0}(0, q) / N_{n}^{0}(0, q)\right)^{1 / 2} .
\end{aligned}
$$

Then clearly $\beta>1$ and by (3.8) $\gamma \geq k$. We now show that

$$
\Phi(s, q) \sim d_{1} s^{\beta} \quad \text { as } s \rightarrow 0^{+}
$$

for some positive constant $d_{1}$. Note that (4.9) with $v=\gamma$ can be written as

$$
\Phi(s, q)=s^{\beta} \exp \left(\int(R(s, q)-R(0, q)) / s d s\right),
$$

where we used $(4.15 \mathrm{~d}, \mathrm{~g})$. Now using (3.9) one can easily check that the function $(R(s, q)-R(0, q)) / s$ is continuous in $[0,1]$. Thus the result $(4.16 \mathrm{a})$ follows. Similarly one can show that

$$
\begin{array}{ll}
C(s, q) \sim d_{2} s^{k-1} & \text { as } s \rightarrow 0^{+}, \\
f(s, q) \sim d_{3} s^{1-\beta} & \text { as } s \rightarrow 0^{+}, \\
\Theta(s, q) \sim d_{4} s^{2-\beta} & \text { as } s \rightarrow 0^{+}, \\
c(s, q) \sim d_{5} s^{k-\beta-1} & \text { as } s \rightarrow 0^{+},
\end{array}
$$

where the $d$ 's are constants and $k$ is as in (3.8). From (4.16d) and $\beta>1$ it follows that $1 / \Theta(s, q)$ belongs to $L^{1}(0,1)$. Also $(4.16 \mathrm{e})$ and $(4.14 \mathrm{a})$ imply that $c(s, q)$ belongs to $L^{1}(0,1)$ and thus the assertion about the regularity of $(4.12)$ follows. 
The material of the plate is called hyperelastic if there exists a twice continuously differentiable function $W(t, n, h, z, m)$ such that

$$
T=W_{t}, \quad N=W_{n}, \quad H=W_{h}, \quad Z=W_{z}, \quad M=W_{m} .
$$

Consequently

$$
T_{n}=N_{t}, \quad Z_{m}=M_{z} .
$$

In this case (3.8) and (4.14) are equivalent to

$$
k<T_{t}^{0}(0, q) / N_{n}^{0}(0, q)<(k-1)^{2} / 4 .
$$

Thus conditions (3.8) and (4.14) are consistent for $k$ sufficiently large when (4.17) holds.

5. An equivalent formulation. Equations $(2.6 \mathrm{a}, \mathrm{b})$ are equivalent to

$$
\begin{gathered}
H^{*}(s) \cos a(s)=-N^{*}(s) \sin a(s), \\
\left(s N^{*}(s) / \cos a(s)\right)^{\prime}=T^{*}(s) .
\end{gathered}
$$

Conditions (2.10a), (2.15), and (2.16) and a global implicit function theorem imply that

$$
(n, h) \rightarrow(N(t, n, h, z, m), H(t, n, h, z, m))
$$

has a thrice continuously differentiable inverse

$$
(N, H) \rightarrow\left(n^{\wedge}(t, N, H, z, m), h^{\wedge}(t, N, H, z, m)\right) .
$$

Define

$$
\begin{gathered}
K(s)=N^{*}(s) / \cos a(s), \\
n^{\#}(t(s), K(s), a(s))=n^{\wedge}(t(s), K(s) \cos a(s), \\
-K(s) \sin a(s), z(s), m(s)), \text { etc., } \\
w^{\#}(s)=\left(t(s), n^{\#}(t(s), K(s), a(s)),\right. \\
\left.h^{\#}(t(s), K(s), a(s)), z(s), m(s)\right), \\
T^{\#}(t(s), K(s), a(s))=T\left(w^{\#}(s)\right), \text { etc. }
\end{gathered}
$$

Hence the BVP of Sec. 2 is equivalent to

$$
\begin{gathered}
(s K(s))^{\prime}=T^{\#}, \\
(s t(s))^{\prime}=n^{\#} \cos a(s)-h^{\#} \sin a(s), \\
\left(s M^{\#}\right)^{\prime}=s\left[h^{\#} \cos a(s)+n^{\#} \sin a(s)\right] K(s) \\
+Z^{\#} \cos a(s), \quad 0<s<1, \\
a(0)=0=a(1), \\
K(1)=-q,\left.\quad s t(s)\right|_{s=0^{+}}=0,
\end{gathered}
$$

where the arguments of $n^{\#}, T^{\#}$, etc. are $(t(s), K(s), a(s))$. We now show that $(5.5,5.6)$ can be reduced to a second-order boundary value problem for the function $a(s)$. 
TheOREM (5.1). Let condition (2.10c) and (3.8) with $k \geq 2$ hold. Hence there exist continuously differentiable functions

$$
K^{\wedge}, t^{\wedge}: C^{1}[0,1] \times[0, \infty) \rightarrow C^{1}[0,1]
$$

such that for any given $(a, q)$ in $C^{1}[0,1] \times[0, \infty)$ with $a$ satisfying $(5.6 \mathrm{a}, \mathrm{b})$, then

$$
\left(K^{\wedge}(a, q)(s), t^{\wedge}(a, q)(s)\right)
$$

is the unique solution of $(5.5 \mathrm{a}, \mathrm{b}),(5.6 \mathrm{c}, \mathrm{d})$, and

$$
K^{\wedge}(a, q)(0)=0, \quad t^{\wedge}(a, q)(0)=1 .
$$

Proof. Define

$$
F: C^{1}[0,1]^{3} \times[0, \infty) \rightarrow C[0,1]
$$

by

$$
F(t, K, a, q)=\left((s K)^{\prime}-T^{\#},(s t)^{\prime}-n^{\#} \cos a+h^{\#} \sin a\right) .
$$

An analysis similar to the one in [3, Sec. 6] based on condition (2.10c) shows that the Fréchet derivative of $F$ with respect to $(t, K)$ is one-to-one and onto. An application now of the implicit function theorem gives the existence of the functions (5.8a).

To get $(5.8 \mathrm{~b})$ we expand the derivatives in $(5.5 \mathrm{a}, \mathrm{b})$ and set $s=0$ to get that

$$
\begin{gathered}
t(0)=n^{\#}(t(0), K(0), a(0))=n(0), \\
K(0)=N^{*}(0)=T\left(w^{\#}(0)\right) .
\end{gathered}
$$

This together with (2.9) imply that

$$
N(t(0), t(0), 0,0,0)=T(t(0), t(0), 0,0,0) \text {. }
$$

Hence by (2.19) and (3.8) we must have $t(0)=1$ and $K(0)=0$.

From this theorem we get that $(5.5,5.6)$ is equivalent to $(5.5 \mathrm{c}),(5.6 \mathrm{a}, \mathrm{b})$, where the arguments of $M^{\#}, h^{\#}$, etc. are now

$$
\left(t^{\wedge}(a, q)(s), K^{\wedge}(a, q)(s), a(s)\right) .
$$

The linearization of this equation about the trivial solution $a=0$ is given by (4.3). This motivates us to write $(5.5 \mathrm{c}),(5.6 \mathrm{a}, \mathrm{b})$ as

$$
L(q) a=F(a, q), \quad a(0)=0=a(1),
$$

where

$$
\begin{gathered}
L(q) a=\left(s M_{m}^{0}(s, q) a^{\prime}(s)+M_{z}^{0}(s, q) a(s)\right)^{\prime} \\
-s N^{0}(s, q)\left[r^{\prime}(s, q)-N^{0}(s, q) / H_{h}^{0}(s, q)\right] a(s) \\
-Z_{z}^{0}(s, q) a(s) / s-Z_{m}^{0}(s, q) a^{\prime}(s), \\
F(a, q)=L(q) a-\left[\left(s M^{\#}\right)^{\prime}-s\left(h^{\#} \cos a(s)+n^{\#} \sin a(s)\right) K(s)-Z^{\#} \cos a(s)\right] .
\end{gathered}
$$

We now proceed exactly as in Sec. 4 to transform the singular operator $L(q)$ into a regular one. Hence using the definitions (4.11), we can make the change of variables

$$
a(s)=s^{\gamma} f(s, q) U(s)
$$


to get that $(5.11)$ is equivalent to

$$
\begin{gathered}
-\left(\Theta(s, q) U^{\prime}(s)\right)^{\prime}+c(s, q) U(s)=F^{\wedge}(U, q), \quad 0<s<1, \\
U(0)=0=U(1),
\end{gathered}
$$

where

$$
F^{\wedge}(U(s), q)=\frac{\Phi(s, q) f(s, q)}{s^{\gamma} s M_{m}^{0}(s, q)} F\left(s^{\gamma} f(s, q) U(s), q\right) .
$$

In Sec. 6 we shall establish the existence of nontrivial solutions of (5.14), and hence for $(5.5,5.6)$, by using a shooting method. For this one needs the existence of solutions of $(5.14 a)$ subject to some appropriate initial conditions. Since the operator to the left of $(5.14 \mathrm{a})$ is regular under the hypotheses of Theorem (4.1), one gets the existence of such solutions by means of a fixed point theorem. Note however that this result does not follow from the usual existence theory (see Naimark [15]) because of the functional dependence of $(5.12 b)$ on $(a, q)$ through (5.8).

6. The existence of buckled states. Let $g$ be a given real number and consider the initial value problem:

$$
\begin{gathered}
-\left(\Theta(s, q) U^{\prime}(s)\right)^{\prime}+c(s, q) U(s)=F^{\wedge}(U, q), \quad 0<s<1, \\
U(0)=0,\left.\quad \Theta(s, q) U^{\prime}(s)\right|_{s=0^{+}}=g .
\end{gathered}
$$

From (4.16) and (5.13) we get that

$$
\begin{gathered}
a^{\prime}(s)=\Theta(s, q)\left[c_{1}(s, q) U(s) / s+c_{2}(s, q) U^{\prime}(s)\right], \\
a(s) / s=c_{3}(s, q) \Theta(s, q) U(s) / s,
\end{gathered}
$$

where $c_{i}(s, q)$ is continuous in $[0,1]$ and

$$
c_{i}(s, q) \sim d_{i} s^{\gamma-1} \quad \text { as } s \rightarrow 0^{+}, i=1,2,3 .
$$

Since by (3.8) we get $\gamma \geq k$, hence from (6.2) we get that for some constants $C$ and $D$ depending on $q$ the following hold:

$$
\begin{gathered}
\left\|a^{\prime} ; C[0,1]\right\| \leq C\left[\|\Theta(\cdot, q) U / s ; C[0,1]\|+\left\|\Theta(\cdot, q) U^{\prime} ; C[0,1]\right\|\right], \\
\|a / s ; C[0,1]\| \leq D\|\Theta(\cdot, q) U / s ; C[0,1]\| .
\end{gathered}
$$

We define

$$
\begin{gathered}
G(q, g) U=\int_{0}^{s}\left\{g+\int_{0}^{t}\left\{c\left(t^{\prime}, q\right) U\left(t^{\prime}\right)-F^{\wedge}\left(U\left(t^{\prime}\right), q\right)\right\} d t^{\prime}\right\} / \Theta(t, q) d t, \\
G^{\prime}(q, g) U=(d / d s)[G(q, g) U] .
\end{gathered}
$$

It follows now that $U$ is a solution of $(6.1)$ if and only if

$$
U=G(q, g) U .
$$

Let $W^{1,1}(0,1)$ be the space of absolutely continuous functions on $(0,1)$. Motivated by the inequalities (6.3) we define the normed linear space

$$
\begin{gathered}
X=\left\{U \text { in } W^{1,1}(0,1): \Theta(s, q) U^{\prime} \text { belongs to } C[0,1], U(0)=0\right\}, \\
\|U ; X\|=\|U ; C[0,1]\|+\left\|\Theta(\cdot, q) U^{\prime} ; C[0,1]\right\|+\|\Theta(\cdot, q) U / s ; C[0,1]\| .
\end{gathered}
$$


Lemma (6.1). $X$ is a Banach space.

Proof. Let $\left\{w_{k}\right\}$ be a Cauchy sequence in $X$. Then the sequences

$$
\left\{w_{k}\right\}, \quad\left\{\boldsymbol{\Theta}(\cdot, q) w_{k}^{\prime}\right\}, \quad\left\{\boldsymbol{\Theta}(\cdot, q) w_{k} / s\right\}
$$

are Cauchy sequences in $C[0,1]$. Hence there exist continuous functions $v, w$, and $z$ such that

$$
\begin{array}{rlrl}
w_{k} & \rightarrow v & & \text { uniformly, } \\
\Theta(\cdot, q) w_{k}^{\prime} \rightarrow w & & \text { uniformly, } \\
\Theta(\cdot, q) w_{k} / s \rightarrow z & & \text { uniformly. }
\end{array}
$$

Let $u_{k}=\Theta(\cdot, q) w_{k}^{\prime}$ so that

$$
w_{k}^{\prime}=u_{k} / \Theta(\cdot, q), \quad u_{k} \rightarrow w \quad \text { uniformly } .
$$

Hence

$$
w_{k}(s)=\int_{0}^{s} w_{k}^{\prime}(t) d t=\int_{0}^{s} u_{k}(t) / \Theta(t, q) d t
$$

Thus for fixed $s$,

$$
\begin{aligned}
\left|w_{k}(s)-\int_{0}^{s} w(t) / \Theta(t, q) d t\right| & =\left|\int_{0}^{s}\left[u_{k}(t)-w(t)\right] / \Theta(t, q) d t\right| \\
& \leq\left\|u_{k}-w ; C[0,1]\right\| \int_{0}^{1} d t / \Theta(t, q) \rightarrow 0
\end{aligned}
$$

as $k \rightarrow \infty$, by $(6.8 \mathrm{e})$ and because $1 / \Theta(\cdot, q)$ is integrable. But $w_{k}(s) \rightarrow v(s)$ as $k \rightarrow \infty$. Hence

$$
v(s)=\int_{0}^{s} w(t) / \Theta(t, q) d t \quad \text { for all } s .
$$

Thus $v$ belongs to $X$ and $w=\Theta(\cdot, q) v^{\prime}$. In a similar way one proves that $z=$ $\Theta(\cdot, q) v / s$.

From (4.16) and (5.15) we get that for some continuous function $c_{4}(s, q)$ we have that

$$
F^{\wedge}(U(s), q)=c_{4}(s, q) s^{-\gamma} F\left(s^{\gamma} f(s, q) U(s), q\right) .
$$

Lemma (6.2). Let condition (3.8) hold with $k \geq 2$. Hence there exist continuous functions $P$ and $Q$ of $\left(w(s), w_{0}(s)\right)$ (cf. (2.1), (4.1)) such that

$$
F(a, q)=s\left[P\left(w(s), w_{0}(s)\right) a(s) / s+Q\left(w(s), w_{0}(s)\right) a^{\prime}(s)\right] .
$$

Proof. From (2.7a), $(2.7 \mathrm{~d})$, and $(5.8 \mathrm{~b})$ it follows that $w(0)=w_{0}(0)$. Hence, since $w$ and $w_{0}$ belong to $C^{1}[0,1]$ for $k \geq 2$, by Taylor's Theorem

$$
w(s)-w_{0}(s)=s d(s),
$$

for some continuous (vector-valued) function of $s$. If we expand about $w_{0}(s)$ the functions of $w(s)$ appearing in $F$ as defined by $(5.12 \mathrm{~b})$, then one gets that $F$ is a sum of terms of the form:

(continuous function of $\left.\left(w(s), w_{0}(s)\right)\right)$ $\times$ (quadratic in the components of $\left.w(s)-w_{0}(s)\right)$. 
Now using (2.11), (2.12), and (2.13) one can show that each term of $(6.11 \mathrm{~b})$ can be written as

$$
\begin{aligned}
& \text { (continuous function of } \left.\left(w(s), w_{0}(s)\right)\right) \\
& \quad \times\left(\text { a component of } w(s)-w_{0}(s)\right) \times\left(a(s) / s \text { or } a^{\prime}(s)\right)
\end{aligned}
$$

(see [3] or [17]). Combining (6.11a) with (6.11c) we get (6.10).

If we now combine $(6.2),(6.9)$, and $(6.10)$ we get that

$$
F^{\wedge}(U, q)=c_{5}(s, q) \Theta(s, q)\left[P^{*}\left(w(s), w_{0}(s)\right) U(s) / s+Q^{*}\left(w(s), w_{0}(s)\right) U^{\prime}(s)\right],
$$

where $c_{5}, P^{*}$, and $Q^{*}$ are continuous functions. This result and the definitions (6.4) show that

$$
G(q, g): X \rightarrow X,
$$

and that there exists a constant $K$ depending on $q$ such that

$$
\|G(q, g) U ; X\| \leq K(|g|+\|U ; X\|) .
$$

Lemma (6.3). The operator $G$ is continuous and compact from $X$ into itself.

Proof. Let $\left\{U_{k}\right\}$ be a bounded sequence in $X$. Hence combining (6.3), (6.4), (6.12), and (6.14) we get that the sequences

$$
\left\{G(q, g) U_{k}\right\}, \quad\left\{\Theta(\cdot, q) G^{\prime}(q, g) U_{k}\right\}, \quad\left\{s^{-1} \Theta(\cdot, q) G(q, g) U_{k}\right\}
$$

are uniformly bounded and equicontinuous on $[0,1]$. The Arzela-Ascoli Theorem implies now that for some continuous functions $v, w$, and $z$

$$
\begin{array}{rlr}
G(q, g) U_{k} \rightarrow v & \text { uniformly, } \\
\Theta(\cdot, q) G^{\prime}(q, g) U_{k} \rightarrow w & \text { uniformly, } \\
s^{-1} \Theta(\cdot, q) G(q, g) U_{k} \rightarrow z & \text { uniformly, }
\end{array}
$$

for some subsequences. The argument now is similar to Lemma (6.1) and one concludes that $w=\Theta(\cdot, q) v^{\prime}, z=\Theta(\cdot, q) v / s$, and hence that $G$ is compact. Now the inequalities (6.3) and the continuity of the functions (5.8a) on $a$ imply that the functions $P^{*}$ and $Q^{*}$ in (6.12) are continuous functions of $U$ in the norm of $X$. The continuity of $G$ now can be obtained from (6.4) and (6.12).

Lemma (6.4). If $g=0$, then $U=0$ is the only solution of (6.1).

Proof. We know that a solution of (6.1) with $g=0$ must satisfy

$$
U=G(q, 0) U .
$$

We shall denote by $X_{s}$ the space defined by $(6.6),(6.7)$ using the interval $[0, s]$ instead of $[0,1]$. An analysis similar to the one leading to $(6.14)$ shows that $(6.16 \mathrm{a})$ implies that for $s^{\prime}$ sufficiently small there exists a constant $C, 0<C<1$, such that

$$
\left\|U ; X_{s^{\prime}}\right\| \leq C\left\|U ; X_{s^{\prime}}\right\| \text {. }
$$

Hence $U=0$ on $\left[0, s^{\prime}\right]$ and now by the usual existence and uniqueness theorem we can conclude that $U=0$ on $[0,1]$. 
We now have:

TheOREM (6.5). For a given $q \geq 0$, the initial value problem (6.1) has connected sets $C^{+}$and $C^{-}$of solution pairs $(U, g)$ which are unbounded respectively on $X \times[0, \infty)$ and $X \times(-\infty, 0]$, and contain $(U=0, g=0)$.

Proof. The result follows from Lemmas (6.3), (6.4) and from Theorem (3.2) of Rabinowitz [18].

We let the function (4.5b) depend also on a positive parameter $d$ which will be a measure of the shear at the center of the plate. Hence we will have the function (4.11c) depending on $d$ also (not (4.11b)). Now Theorem (6.5) still holds for any given $d>0$. We shall denote a solution of $(6.1)$ for a given triple $(q, g, d)$ by $U^{*}(s, q, g, d)$. Using the Implicit Function Theorem one can show that this solution is smooth in $(q, g, d)$ in a neighborhood of any $\left(q^{\prime}, 0, d^{\prime}\right)$. Henceforth we shall assume this property. Now the BVP $(5.14)$ is equivalent to

$$
U^{*}(1, q, g, d)=0 \text {. }
$$

Note that the symmetry conditions $(2.11),(2.12)$, and (2.13) imply that

$$
U^{*}(1, q,-g, d)=-U^{*}(1, q, g, d) \text {. }
$$

We now study the behavior of the solution set of $(6.17)$ in a neighborhood of certain singular points.

Note that by Lemma (6.4)

$$
U^{*}(s, q, 0, d)=0 .
$$

Since the linearization of (6.1a) about $U=0$ is given by (4.12a), it follows from (6.1) that $U_{g}^{*}(s, q, 0, d)$ is a solution of the initial value problem

$$
\begin{gathered}
-\left(\Theta(s, q) U^{\prime}(s)\right)^{\prime}+c(s, q, d) U(s)=0, \\
U(0)=0,\left.\quad \Theta(s, q) U^{\prime}(s)\right|_{s=0^{+}}=1 .
\end{gathered}
$$

Hence the equation

$$
U_{g}^{*}(1, q, 0, d)=0
$$

is the characteristic equation defining the eigenpairs $\left(q^{*}, d^{*}\right)$ of Theorem (4.1). Thus if $\left(q^{*}, d^{*}\right)$ is a solution of $(6.21)$ and we further assume that

$$
\begin{gathered}
U_{g q}^{*}\left(1, q^{*}, 0, d^{*}\right)=0, \\
U_{g d}^{*}\left(1, q^{*}, 0, d^{*}\right) \neq 0, \\
U_{g q q}^{*}\left(1, q^{*}, 0, d^{*}\right) \neq 0,
\end{gathered}
$$

then the symmetry condition (6.18) allows us to invoke the theory in Golubitsky and Schaeffer [8] to get:

TheOREM (6.6). Let $\left(q^{*}, d^{*}\right)$ satisfy conditions (6.21) and (6.22). Then $(q, g, d) \rightarrow$ $U^{*}(1, q, g, d)$ is a $Z_{2}$-universal unfolding of $(q, g) \rightarrow U^{*}\left(1, q, g, d^{*}\right)$. For $(q, g, d)$ near $\left(q^{*}, 0, d^{*}\right)$ the bifurcation diagram of $(6.17)$ is strongly equivalent to $x^{3}-e^{2} x+f x=0$, where $(x, e, f)$ corresponds to $\left(g, q-q^{*}, d-d^{*}\right)$. 
7. Numerical examples. In this section we present two numerical examples for which the conditions of Theorem (6.6) are satisfied. First we shall consider a circular plate made up of an isotropic material. In this case it can be shown that (see [3])

$$
\begin{aligned}
& N(t, n, 0, z, m)=T(n, t, 0, m, z), \\
& M(t, n, 0, z, m)=Z(n, t, 0, m, z),
\end{aligned}
$$

and thus (setting $t=n, z=m=0$ ) that (3.4) holds with equality. It follows that the solution of $(3.2),(3.3)$ is given by $r(s)=t(q) s$, where $t(q)$ is the unique solution of

$$
N(t, t, 0,0,0)+q=0 .
$$

The results of Secs. 4, 5, and 6 do not directly apply in this case. However because of the special form of $r(s)$ and by using (7.1) one can explicitly construct the mappings appearing in (6.21) and (6.22) and check the conditions in the theorem.

We assume that the function $H$ is linear in $h$ and independent of $(t, n, z, m)$, i.e.,

$$
H(t, n, h, z, m)=d h,
$$

form some constant $d>0$. Note that (7.3) is compatible with $(2.10 \mathrm{a}, \mathrm{c}),(2.11 \mathrm{~b})$, $(2.12 \mathrm{c})$, and (2.16). We emply the notation

$$
G^{0}(q)=G(t(q), t(q), 0,0,0),
$$

for any constitutive function $N, T, N_{t}$, etc. It follows now from (7.1) that (4.3) simplifies to

$$
\begin{gathered}
M_{m}^{0}(q)\left(s p^{\prime}(s)\right)^{\prime}=s N^{0}(q)\left[t(q)-N^{0}(q) / d\right] p(s)+Z_{z}^{0}(q) p(s) / s, \\
p(0)=0=p(1) .
\end{gathered}
$$

From [20] or [11] we get that (7.5) has nontrivial solutions provided

$$
\begin{gathered}
f\left(q^{*}, d^{*}\right)=j_{k}^{2}, \quad k \geq 0, \\
f(q, d)=N^{0}(q)\left[N^{0}(q) / d-t(q)\right] / M_{m}^{0}(q),
\end{gathered}
$$

where the $\left\{j_{k}\right\}$ are the positive roots of $J_{1}$ (the Bessel function of the first kind of order one). Thus (7.6a) is the equation corresponding to (6.21). It follows now that (6.22) is equivalent to

$$
f_{q}\left(q^{*}, d^{*}\right)=0, \quad f_{d}\left(q^{*}, d^{*}\right) \neq 0, \quad f_{q q}\left(q^{*}, d^{*}\right) \neq 0 .
$$

This implies that the curve of eigenpairs (7.6a) has a turning point in the variable $d$ on the $(q, d)$-plane.

For a particular example we take

$$
\begin{array}{cl}
N(t, n)=-n^{-\alpha}+1, & T(t, n)=N(n, t), \\
M_{m}^{0}(q)=\mu N_{n}^{0}(q) & (\text { cf. }(4.15 \mathrm{c})),
\end{array}
$$


for some positive constants $\alpha$ and $\mu$. (Henceforth we assume that $d_{0}(s)$ in $(4.15 \mathrm{c}$ ) is constant. For conditions that ensure this see [3]). In this case one easily finds that the solution of (7.2) is given by

$$
t(q)=(1+q)^{-1 / \alpha} .
$$

Thus (7.6) reduces to

$$
(q / \mu \alpha)\left(q / d+(1+q)^{-1 / \alpha}\right)(1+q)^{-(\alpha+1) / \alpha}-j_{k}^{2}=0 .
$$

Note that the second condition in (7.7) clearly holds. The first condition in (7.7) is equivalent to

$$
2 q-((\alpha+1) / \alpha) q^{2} /(1+q)=d((1+2 / \alpha)(q /(1+q))-1)(1+q)^{-1 / \alpha} .
$$

By studying the graphs of the functions on both sides of this equation one finds that

(i) if $\alpha<1$, then for a given $d>0$ Eq. (7.11) always has a solution $q>0$;

(ii) if $\alpha>1$ and $d>0$ is sufficiently large, then (7.11) has a solution $q>0$.

If we let $\left(q^{*}, d^{*}\right)$ denote the solution of (7.11) for a given $\alpha$, then Eq. (7.10) is satisfied by choosing $\mu$ appropriately. Physically one can interpret the case $\alpha<1$ as that of a soft material (in the radial direction) and $\alpha>1$ as a hard or strong material (in the radial direction). Thus the results above, for the family of isotropic materials (7.8), show that nonbifurcating buckled states of the plate exist for soft materials with appropriate bending moments and for hard materials with appropriate bending moments and shear response.

We now construct an example of an anisotropic material. We assume that (7.3) holds and that

$$
\begin{gathered}
N(t, n)=-n^{-\alpha}, \quad T(t, n)=-B t^{-\alpha}, \\
M_{m}^{0}(s, q)=\mu N_{n}^{0}(s, q),
\end{gathered}
$$

where $B, \mu>0$ and $\alpha>1$. One can show now that the solution of (3.2), (3.3) is given by

$$
r(s)=K s^{\delta}
$$

where $K$ and $\delta$ satisfy

$$
1-\alpha(\delta-1)=B \delta^{\alpha}, \quad K=\left(\delta q^{1 / \alpha}\right)^{-1} .
$$

(Equation (7.14a) has a solution which is unique for any $B>0$ and $\alpha>1$.) It follows now that (4.4) reduces to

$$
\begin{aligned}
p^{\prime \prime}(s) & +\left(B \delta^{\alpha}+1-\delta\right) p^{\prime}(s) / s \\
+ & {\left[q^{-1 / \alpha} s^{\delta}\left(q^{-1 / \alpha} s^{\delta}+(q / d) s^{1-\alpha(\delta-1)}\right) / \alpha \mu-B \delta^{\alpha+1}\right] p(s) / s^{2}=0, }
\end{aligned}
$$

subject to $(4.3 \mathrm{~b}, \mathrm{c})$. Although we are not able to solve this equation explicitly in terms of any special function, we show that $(q, d, \mu)$ can be chosen such that $(6.21),(6.22)$ hold. 
When (7.12) holds, we get that (4.9) and (4.11) reduce to

$$
\begin{gathered}
\Phi(s)=s^{e+1}, \quad e=B \delta^{\alpha}+\delta, \\
f(s)=1+\left(s^{-e}-1\right) / e, \quad \Theta(s)=s^{e+1} f^{2}(s), \\
c(s, q, d, \mu)=q^{-1 / \alpha} s^{\delta+e-1} f^{2}(s)\left(q^{-1 / \alpha} s^{\delta}+(q / d) s^{1-\alpha(\delta-1)}\right) / \alpha \mu .
\end{gathered}
$$

If we let $U(s, q, d, \mu)$ be the solution of $(6.20)$ corresponding to $(7.16 \mathrm{c}, \mathrm{d})$, then (6.21) is equivalent to

$$
U(1, q, d, \mu)=0
$$

and (6.22) becomes

$$
\begin{aligned}
& \int_{0}^{1} c_{q}(s, q, d, \mu) U^{2}(s, q, d, \mu) d s=0, \\
& \int_{0}^{1} c_{d}(s, q, d, \mu) U^{2}(s, q, d, \mu) d s \neq 0 .
\end{aligned}
$$

From $(7.16 \mathrm{~d})$ one easily checks $(7.18 \mathrm{~b})$. To get $(7.18 \mathrm{a})$ we assume that

$$
\|U(\cdot, q, d, \mu) ; C[0,1]\| \leq D,
$$

where $D$ is independent of $(q, d, \mu)$. (See Naimark [15, Part I] for a discussion on the asymptotic behavior of eigenfunctions in which (7.19) holds under mild conditions.) Since $\alpha>1$, one can see from $(7.16 \mathrm{~d})$ that $c_{q}$ alternates signs on $[0,1]$ and one can control these sign changes by varying $(q, d)$. It follows from this and (7.19) that in principle one can adjust $(q, d)$ such that $(7.18 \mathrm{a})$ holds. Once $(q, d)$ have been chosen in this way, with them fixed one can treat $\mu$ in (7.17) like an eigenvalue parameter and get conditions for the existence of $\mu$ 's satisfying (7.17). Although this construction of $(q, d, \mu)$ satisfying (7.17), (7.18) is not explicit, it shows that under reasonable constitutive assumptions one can except (6.21), (6.22) to hold for anisotropic materials with appropriate shear and bending moments.

8. Conclusions. The results of Theorem (6.6) show that a certain shear parameter can be used to construct a universal unfolding of the solution set of the boundary value problem of Sec. 2 near singular points satisfying conditions (6.21), (6.22). In a sense this captures all possible perturbations of the bifurcating set in a neighborhood of these points. The key idea here was the reduction of the original boundary value problem to the scalar equation (6.21). This is essentially the Liapunov-Schmidt reduction method. When conditions (6.22) are not easily verifiable, one can check them numerically by constructing the solution curve (6.21) using path following techniques. (See Cliffe and Spence [6] for an application of this method to the Taylor problem in fluid mechanics.) One can in principle use this method to study the effects of variations in other constitutive parameters on the solution set.

One can also get corresponding global results by employing the multi-parameter global bifurcation results of Alexander and Antman [1]. In fact, the results of NegronMarrero and Antman [17] show that the boundary value problem of Sec. 2 can be recast in the form

$$
u=L(q, d) u+K(q, d, u),
$$


where $L(q, d)$ is a linear and compact operator on some appropriate Banach space, $K(q, d, \cdot)$ is compact and continuous, and $K(q, d, u)=o(\|u\|)$ as $\|u\| \rightarrow 0$ uniformly for $(q, d)$ in compact subsets of $R^{2}$. Conditions (6.21), (6.22) imply that $d^{*}$ is a simple eigenvalue (in the sense of Magnus [14]) of $L\left(q^{*}, d\right)$ and thus Corollary (2.50) of Alexander and Antman [1] can be applied to get the global existence of a connected set of nontrivial solutions bifurcating from $\left(q^{*}, d^{*}, 0\right)$ and having topological dimension greater than two at each point. See Antman and Pierce [4] for the details of this argument in the case the structure is a rod.

Acknowledgment. This research was supported by the National Science Foundation under Grant No. DMS-8722521.

\section{REFERENCES}

[1] J. C. Alexander and S. S. Antman, Global behavior of bifurcating multidimensional continua of solutions for multiparameter nonlinear eigenvalue problems, Arch. Rational Mech. Anal. 76, 339354 (1981)

[2] J. C. Alexander and S. S. Antman, Global behavior of solutions of nonlinear equations depending on infinite-dimensional parameters, Indiana Univ. Math. J. 32, 39-62 (1983)

[3] S. S. Antman, Buckled states of nonlinearly elastic plates, Arch. Rational Mech. Anal. 67, 111-149 (1978)

[4] S. S. Antman and J. F. Pierce, The intricate global structure of buckled states of compressible columns, SIAM J. Appl. Math. 50, 395-419 (1990)

[5] S. S. Antman and P. V. Negron-Marrero, The remarkable nature of radially symmetric equilibrium states of aelotropic nonlinearly elastic bodies, J. Elasticity 18, 131-164 (1987)

[6] K. A. Cliffe and A. Spence, The calculation of high order singularities in the finite Taylor problem, Proc. Conf. on Numerical Methods for Bifurcation Problems (Dortmund, August 22-26, 1983), Birkhaüser, Basel, 1984

[7] M. G. Crandell and P. H. Rabinowitz, Nonlinear Sturm-Liouville eigenvalue problems and topological degree, J. Math. Mech. 19, 1083-1102 (1970),

[8] M. Golubitsky and D. Schaeffer, Singularities and Groups in Bifurcation Theory, Vol. I, SpringerVerlag, New York, 1985

[9] T. J. Healey, Global bifurcation and continuation in the presence of symmetry with an application to solid mechanics, SIAM J. Math. Anal. 19, 824-840 (1988)

[10] T. J. Healey, Symmetry and equivariance in nonlinear elastostatics. I, Arch. Rational Mech. Anal. 105, 205-228 (1989)

[11] E. Jahnke and F. Emde, Tables of Functions-With Formulas and Curves, 4th ed., Dover, New York, 1945

[12] H. G. Kaper, M. K. Kwong, and A. Zettl, Regularizing transformations for certain singular SturmLiouville boundary value problems, SIAM J. Math. Anal. 15, 957-963 (1984)

[13] J. Leray and J. Schauder, Topologie et equations fonctionelles, Ann. Sci. Ecole Norm. Sup. (3) 51, 45-78 (1934)

[14] R. J. Magnus, A generalization of multiplicity and the problem of bifurcation, Proc. London Math. Soc. (3) 32, 251-278 (1976)

[15] M. A. Naimark, Linear Differential Operators. I, II, Ungar, New York, 1968

[16] P. V. Negron-Marrero, Necked states of nonlinearly elastic plates, Proc. Roy. Soc. Edinburgh, Sect. A 112, 277-291 (1989)

[17] P. V. Negron-Marrero and S. S. Antman, Singular global bifurcation problems for the buckling of anisotropic plates, Proc. Roy. Soc. London Ser. A 427, 95-137 (1990)

[18] P. H. Rabinowitz, Some global results for nonlinear eigenvalue problems, J. Funct. Anal. 7, 487-513 (1971)

[19] D. H. Sattinger, Branching in the presence of symmetry, CBMS-NSF Regional Conf. Ser. in Appl. Math., vol. 40, SIAM, Philadelphia, 1983

[20] G. N. Watson, A Treatise on the Theory of Bessel Functions, 2nd Ed., Cambridge Univ. Press, 1962 